\title{
ON MOROZOV'S DISCREPANCY PRINCIPLE FOR NONLINEAR ILL-POSED EQUATIONS
}

\author{
M. T. NAIR
}

(Received 21 July 2008)

\begin{abstract}
Morozov's discrepancy principle is one of the simplest and most widely used parameter choice strategies in the context of regularization of ill-posed operator equations. Although many authors have considered this principle under general source conditions for linear ill-posed problems, such study for nonlinear problems is restricted to only a few papers. The aim of this paper is to apply Morozov's discrepancy principle for Tikhonov regularization of nonlinear ill-posed problems under general source conditions.
\end{abstract}

2000 Mathematics subject classification: primary 65J20, 65M30.

Keywords and phrases: inverse problems, ill-posed problems, Tikhonov regularization, discrepancy principle.

\section{Introduction}

Many of the inverse problems that appear in science and engineering can be modelled as ill-posed equations of the form

$$
F(x)=y,
$$

where $F: D(F) \subseteq X \rightarrow Y$ is an operator (not necessarily linear) between Hilbert spaces $X$ and $Y$ (see, for example, [1]). We assume that the operator $F$ and the data $y$ are such that the above equation has a solution $\widehat{x}$ which minimizes the function $x \mapsto\|x-\bar{x}\|$, where $\bar{x}$ is an initial guess at the unknown solution. As the data available is often noisy, say $y^{\delta}$ in place of $y$ with

$$
\left\|y-y^{\delta}\right\| \leq \delta
$$

for known noise level $\delta>0$, the ill-posedness of the equation demands application of certain regularization methods for obtaining stable approximate solutions. Tikhonov regularization is one of the most widely used such methods. In Tikhonov regularization, one looks for a minimizer $x_{\alpha}^{\delta}$ of the function

$$
x \mapsto J_{\alpha}\left(x, y^{\delta}\right):=\left\|F(x)-y^{\delta}\right\|^{2}+\alpha\|x-\bar{x}\|^{2},
$$

where $\alpha>0$ is a parameter to be chosen appropriately.

(C) 2009 Australian Mathematical Society 0004-9727/2009 \$16.00 
It is known (see [1]) that if $F$ is weakly closed, then for every $(y, \bar{x}) \in Y \times X$ and for every $\alpha>0$, there exists $x \in D(F)$ such that

$$
J_{\alpha}(x, y)=\inf _{u \in D(F)} J_{\alpha}(u, y) .
$$

Suppose that $D(F)$ contains a neighbourhood of $\widehat{x}$, and $F$ is Fréchet differentiable at $\widehat{x}$. It is known that if $\widehat{x}$ belongs to a source set of the form

$$
M_{\nu, \rho}:=\left\{\left(A^{*} A\right)^{v} v:\|v\| \leq \rho\right\}
$$

for some $\rho>0$ and $0<v \leq 1$, with $A:=F^{\prime}(\widehat{x})$, the Fréchet derivative of $F$ at $\widehat{x}$, then by choosing $\alpha$ either a priori as $\alpha:=c_{0} \delta^{2 /(2 v+1)}$ or by a Morozov-type discrepancy principle in which $\alpha$ satisfies the inequality

$$
c_{1} \delta \leq\left\|F\left(x_{\alpha}^{\delta}\right)-y^{\delta}\right\| \leq c_{2} \delta
$$

with $c_{2} \geq c_{1} \geq 1$, then we obtain the 'optimal estimate'

$$
\left\|\widehat{x}-x_{\alpha}^{\delta}\right\|=O\left(\delta^{2 v /(2 v+1)}\right) .
$$

But there are many examples of inverse problems where the requirement that $\widehat{x}$ belongs to $M_{v, \rho}$ becomes too restrictive (see, for example, [5, 6, 14]). A general form of source set which was found convenient and useful in many linear ill-posed problems is

$$
M_{\varphi, \rho}:=\left\{\sqrt{\varphi\left(A^{*} A\right)} v:\|v\| \leq \rho\right\},
$$

where $\varphi$ is a suitable function which is general enough to include many of the standard source conditions, including the Hölder type in which $\varphi(\lambda):=\lambda^{\nu}$ for $0<\nu \leq 1$, and the logarithmic type in which $\varphi(\lambda):=[\ln (1 / \lambda)]^{-p}$ for $p>0$.

The general source condition in the context of linear ill-posed problems has been considered extensively in recent years (see, for example, [10-14, 17]). Extensions of such source conditions to nonlinear problems have also been considered (see, for example, [7, 8]). However, the general source condition combined with Morozov's discrepancy principle, one of the most widely used parameter choice strategies in the context of linear ill-posed equations, does not seem to have been studied for nonlinear problems.

The aim of this short paper is to obtain an order optimal estimate for the error involved in nonlinear ill-posed equations under a general source condition, along the lines of the analysis in [13], by choosing the regularization parameter $\alpha$ according to the Morozov-type discrepancy principle (1.3).

We may observe that if $A$ is injective and $\varphi$ is an index function, that is, if $\varphi$ is a positive monotonically increasing, continuous function defined on a suitable interval $[0, a]$ satisfying $\lim _{\lambda \rightarrow 0} \varphi(\lambda)=0$, then the source set $M_{\varphi, \rho}$ defined above can be thought of as a closed ball of radius $\rho$ in a Hilbert space $X_{\varphi}$ which is the completion of the subspace

$$
D_{\varphi}:=\left\{x \in X: \exists v_{x} \in X \text { with } x=\sqrt{\varphi\left(A^{*} A\right) v_{x}}\right\}
$$


with respect to the norm

$$
\|x\|_{\varphi}:=\left\|v_{x}\right\|, \quad x \in D_{\varphi} .
$$

The variable Hilbert scale $\left\{X_{\varphi}: \varphi\right.$ an index function $\}$ was considered first by Hegland (see [3, 4]) as a generalization of the concept of Hilbert scale.

In this short paper we consider such general source condition and use Morozov's discrepancy principle (1.3) as the parameter choice strategy for choosing the regularization parameter $\alpha$.

\section{The main result}

The following main theorem of this paper extends the known result (see [13]) for linear ill-posed problems to the nonlinear case. We shall make use of the following assumptions.

ASSUMPTION 2.1. (a) The exact data $y$ belongs to $R(F)$, the range of $F$, and $\widehat{x} \in D(F)$ is such that $F(\widehat{x})=y$.

(b) The operator $F$ is Fréchet differentiable at $\widehat{x}$ and there exists $\eta>0$ such that

$$
\left\|F^{\prime}(\widehat{x})(\widehat{x}-x)\right\| \leq \eta\|F(\widehat{x})-F(x)\| \quad \forall x \in B_{r}(\widehat{x}) ; \quad r \geq\|\widehat{x}-\bar{x}\| .
$$

(c) There exist $c_{1}, c_{2}$ with $c_{2} \geq c_{1} \geq 1$ such that for every $\delta>0$, there exists $\alpha:=$ $\alpha\left(\delta, y^{\delta}\right)$ satisfying

$$
c_{1} \delta \leq\left\|F\left(x_{\alpha}^{\delta}\right)-y^{\delta}\right\| \leq c_{2} \delta .
$$

(d) There exists $\rho>0$ such that

$$
\widehat{x}-\bar{x} \in\left\{\sqrt{\varphi\left(A^{*} A\right)} v:\|v\| \leq \rho\right\}
$$

where $A:=F^{\prime}(\widehat{x})$ and $\varphi:[0, a] \rightarrow[0, \infty)$ with $a \geq\|A\|^{2}$ is a monotonically increasing, continuous and concave function satisfying $\lim _{\lambda \rightarrow 0} \varphi(\lambda)=0$.

We now state and prove the main theorem of this paper.

THEOREM 2.2. Under Assumption 2.1,

$$
\left\|\widehat{x}-x_{\alpha}^{\delta}\right\| \leq c_{0} \sqrt{\psi^{-1}\left(\delta^{2} / \rho^{2}\right)}
$$

where $c_{0}:=\max \left\{2, \eta\left(1+c_{2}\right)\right\}$ and $\psi(\lambda):=\lambda \varphi^{-1}(\lambda)$ for $\lambda \in[0, a]$.

PROOF. By the definition of $x_{\alpha}^{\delta}$ and by the fact that $y=F(\widehat{x})$, we have

$$
\begin{aligned}
\delta^{2}+\alpha\left\|x_{\alpha}^{\delta}-\bar{x}\right\|^{2} & \leq c_{1} \delta^{2}+\alpha\left\|x_{\alpha}^{\delta}-\bar{x}\right\|^{2} \\
& \leq\left\|F\left(x_{\alpha}^{\delta}\right)-y^{\delta}\right\|^{2}+\alpha\left\|x_{\alpha}^{\delta}-\bar{x}\right\|^{2} \\
& \leq\left\|F(\widehat{x})-y^{\delta}\right\|^{2}+\alpha\|\widehat{x}-\bar{x}\|^{2} \\
& \leq \delta^{2}+\alpha\|\widehat{x}-\bar{x}\|^{2} .
\end{aligned}
$$


Hence, $\left\|x_{\alpha}^{\delta}-\bar{x}\right\|^{2} \leq\|\widehat{x}-\bar{x}\|^{2}$. Thus, by Assumption 2.1(d),

$$
\begin{aligned}
\left\|x_{\alpha}^{\delta}-\widehat{x}\right\|^{2} & \leq\|\widehat{x}-\bar{x}\|^{2}+\left\|x_{\alpha}^{\delta}-\widehat{x}\right\|^{2}-\left\|x_{\alpha}^{\delta}-\bar{x}\right\|^{2} \\
& =2\left\langle x_{\alpha}^{\delta}-\widehat{x}, \widehat{x}-\bar{x}\right\rangle \\
& =2\left\langle x_{\alpha}^{\delta}-\widehat{x}, \sqrt{\varphi\left(A^{*} A\right)} v\right\rangle \\
& =2\left\langle\sqrt{\varphi\left(A^{*} A\right)}\left(x_{\alpha}^{\delta}-\widehat{x}\right), v\right\rangle \\
& \leq 2 \rho\left\|\sqrt{\varphi\left(A^{*} A\right)}\left(x_{\alpha}^{\delta}-\widehat{x}\right)\right\| \\
& \leq c_{0} \rho\left\|\sqrt{\varphi\left(A^{*} A\right)}\left(x_{\alpha}^{\delta}-\widehat{x}\right)\right\| .
\end{aligned}
$$

Therefore,

$$
\frac{\left\|x_{\alpha}^{\delta}-\widehat{x}\right\|^{2}}{c_{0}^{2} \rho^{2}} \leq \frac{\left\|\sqrt{\varphi\left(A^{*} A\right)}\left(x_{\alpha}^{\delta}-\widehat{x}\right)\right\|^{2}}{\left\|x_{\alpha}^{\delta}-\widehat{x}\right\|^{2}} .
$$

Using the spectral representation of the self-adjoint operator $A^{*} A$ (see [9]),

$$
\frac{\left\|\sqrt{\varphi\left(A^{*} A\right)} x\right\|^{2}}{\|x\|^{2}}=\frac{\int_{0}^{a} \varphi(\lambda) d\left\langle E_{\lambda} x, x\right\rangle}{\int_{0}^{a} d\left\langle E_{\lambda} x, x\right\rangle}
$$

and hence, by Jensens's inequality (see [16]),

$$
\varphi^{-1}\left(\frac{\left\|\sqrt{\varphi\left(A^{*} A\right)} x\right\|^{2}}{\|x\|^{2}}\right) \leq \frac{\int_{0}^{a} \lambda d\left\langle E_{\lambda} x, x\right\rangle}{\int_{0}^{a} d\left\langle E_{\lambda} x, x\right\rangle}=\frac{\|A x\|^{2}}{\|x\|^{2}} .
$$

Thus,

$$
\varphi^{-1}\left(\frac{\left\|x_{\alpha}^{\delta}-\widehat{x}\right\|^{2}}{c_{0}^{2} \rho^{2}}\right) \leq \varphi^{-1}\left(\frac{\left\|\sqrt{\varphi\left(A^{*} A\right)}\left(x_{\alpha}^{\delta}-\widehat{x}\right)\right\|^{2}}{\left\|x_{\alpha}^{\delta}-\widehat{x}\right\|^{2}}\right) \leq \frac{\left\|A\left(x_{\alpha}^{\delta}-\widehat{x}\right)\right\|^{2}}{\left\|x_{\alpha}^{\delta}-\widehat{x}\right\|^{2}} .
$$

By (a) and (b) in Assumption 2.1,

$$
\left\|A\left(x_{\alpha}^{\delta}-\widehat{x}\right)\right\| \leq \eta\left\|y-F\left(x_{\alpha}^{\delta}\right)\right\| \leq \eta\left(\left\|y-y^{\delta}\right\|+\left\|y^{\delta}-F\left(x_{\alpha}^{\delta}\right)\right\|\right)=\eta\left(\delta+c_{2} \delta\right)=c_{0} \delta .
$$

Thus,

$$
\varphi^{-1}\left(\frac{\left\|x_{\alpha}^{\delta}-\widehat{x}\right\|^{2}}{c_{0}^{2} \rho^{2}}\right) \leq \frac{\left\|A\left(x_{\alpha}^{\delta}-\widehat{x}\right)\right\|^{2}}{\left\|x_{\alpha}^{\delta}-\widehat{x}\right\|^{2}} \leq \frac{c_{0}^{2} \delta^{2}}{\left\|x_{\alpha}^{\delta}-\widehat{x}\right\|^{2}}
$$

so that

$$
\psi\left(\frac{\left\|x_{\alpha}^{\delta}-\widehat{x}\right\|^{2}}{c_{0}^{2} \rho^{2}}\right) \leq \frac{\delta^{2}}{\rho^{2}}
$$

Equivalently, $\left\|x_{\alpha}^{\delta}-\widehat{x}\right\|^{2} \leq c_{0} \rho \sqrt{\psi^{-1}\left(\delta^{2} / \rho^{2}\right)}$. This completes the proof. 
REMARK 2.3.

(i) Well-known special cases for $\varphi$ in Assumption 2.1(d) are $\varphi(\lambda):=\lambda^{v}$ for $0<$ $v \leq 1$ and $\varphi(\lambda):=[\ln (1 / \lambda)]^{-p}$ for $p>0$; the first case corresponds to the so-called mildly ill-posed problems, and second to the exponentially ill-posed problems (see, for example [5, 6]).

(ii) It is known that many of the ill-posed problems that occur in applications satisfy a condition of the form

$$
\left\|F(x)-F(\widehat{x})-F^{\prime}(\widehat{x})(\widehat{x}-x)\right\| \leq \eta_{0}\|F(\widehat{x})-F(x)\|
$$

for all $x \in B_{r}(\widehat{x})$ for some $\eta_{0}>0$. This is known in the literature as the $\eta$-condition ([2]). Clearly, condition (b) in Assumption 2.1 is equivalent to the above $\eta$-condition.

(iii) Ramlau [15] has given sufficient conditions under which the regularization parameter $\alpha$ satisfying

$$
c_{1} \delta \leq\left\|F\left(x_{\alpha}^{\delta}\right)-y^{\delta}\right\| \leq c_{2} \delta
$$

as in Assumption 2.1(d) exists.

\section{Conclusion}

We have applied a Morozov-type discrepancy principle for choosing the regularization parameter in Tikhonov regularization of a nonlinear ill-posed equation. Under suitable assumptions on the nonlinear operator, it is shown that the procedure yields order optimal error estimates under a general source condition.

\section{References}

[1] H. W. Engl, M. Hanke and A. Neubauer, Regularization of Inverse Problems (Kluwer, Dordrecht, 1993).

[2] H. W. Engl, K. Kunisch and A. Neubauer, 'Convergence rates for Tikhonov regularization of nonlinear ill-posed problems', Inverse Problems 5 (1989), 523-540.

[3] M. Hegland, 'An optimal order regularization method which does not use additional smoothness assumptions', SIAM J. Numer. Anal. 29 (1992), 1446-1461.

[4] M. Hegland, 'Variable Hilbert scales and their interpolation inequalities with applications to Tikhonov regularization', Appl. Anal. 59 (1995), 207-223.

[5] T. Hohage, 'Logarithmic convergence rates of the iteratively regularized Gauss-Newton method for an inverse potential and an inverse scattering problem', Inverse Problems 13 (1997), 1279-1299.

[6] _ 'Regularization of exponentially ill-posed problems', Numer. Funct. Anal. Optim. 21 (2000), 439-464.

[7] S. Lu, S. V. Pereverzev and R. Ramlau, 'An analysis of Tikhonov regularization for nonlinear illposed problems under a general smoothness assumption', Inverse Problems 23 (2007), 217-230.

[8] P. Mahale and M. T. Nair, 'Tikhonov regularization of nonlinear ill-posed equations under general source conditon', J. Inverse Ill-Posed Probl. 15 (2007), 813-829.

[9] M. T. Nair, Functional Analysis: A First Course (Prentice-Hall of India, New Delhi, 2002). 
[10] _ 'On improving error estimates for Tikhonov regularization using an unbounded operator', J. Anal. 14 (2006), 143-157.

[11] M. T. Nair and S. Pereverzev, 'Regularized collocation method for Fredholm integral equations of the first kind', J. Complexity 23 (2007), 454-467.

[12] M. T. Nair, S. Pereverzev and U. Tautenhahn, 'Regularization in Hilbert scales under general smoothing conditions', Inverse Problems 21 (2005), 1851-1869.

[13] M. T. Nair, E. Schock and U. Tautenhahn, 'Morozov's discrepancy principle under general source conditions', J. Anal. Anw. 22 (2003), 199-214.

[14] S. Pereverzev and E. Schock, 'Morozov's discrepancy principle for Tikhonov regularization of severely ill-posed problems', Numer. Funct. Anal. Optim. 21 (2000), 901-920.

[15] R. Ramlau, 'Morozov's discrepancy principle for Tikhonov regularization of nonlinear operators', Numer. Funct. Anal. Optim. 23 (2002), 147-172.

[16] W. Rudin, Real and Complex Analysis, 3rd edn (McGraw-Hill, New York, 1987).

[17] U. Tautenhahn, 'Optimality for ill-posed problems under general source conditions', Numer. Funct. Anal. Optim. 19 (1998), 377-398.

M. T. NAIR, Department of Mathematics, I.I.T. Madras, Chennai 600 036, India

e-mail: mtnair@iitm.ac.in 